\title{
Revista Colombiana de

\section{Valoración mediante ecocardiografía bidimensional del área y el volumen de la aurícula derecha indexados en el área de superficie corporal en población sana}

\author{
Juan G. Echeverri, Jannet Mosquera* y Gustavo Restrepo \\ Unidad de Cardiología, Servicio de Ecocardiografía, Clínica Medellín, Medellín, Colombia
}

Recibido el 25 de abril de 2014; aceptado el 10 de junio de 2015

Disponible en Internet el 5 de septiembre de 2015

\section{PALABRAS CLAVE \\ Ecocardiografía; \\ Aurícula derecha; \\ Valores de referencia}

\begin{abstract}
Resumen
Objetivo: Determinar el área y el volumen normal de la aurícula derecha indexándola en la superficie corporal mediante ecocardiografía bidimensional en población sana para contar con estos valores como referente ante estudios futuros de población no sana.

Metodología: Estudio observacional descriptivo.

Resultados: Se incluyeron 64 pacientes sanos, entre los 17 y 68 años de edad, que cumplían los criterios de selección. El promedio de edad fue de 34 años ( $D E \pm 12,19)$ y la razón de masculinidad fue de 1:1,78, encontrándose un promedio del área de la aurícula derecha calculado en $12,2 \mathrm{~cm}^{2}(D E \pm 1,46)$ con un mínimo observado en $10 \mathrm{~cm}^{2}$ y un máximo de $16 \mathrm{~cm}^{2}$. Además de esta medición, para esta cavidad se calculó el volumen, donde se observó un promedio de $28,7 \mathrm{~mL}$, con un mínimo de $19 \mathrm{~mL}$ y un máximo de $42 \mathrm{~mL}(\mathrm{DE} \pm 5,19)$.

Conclusiones: Este es el primer estudio en Colombia que reporta los valores normales indexados en el área de superficie corporal, del área y el volumen de la aurícula derecha en población sana.

(C) 2015 Sociedad Colombiana de Cardiología y Cirugía Cardiovascular. Publicado por Elsevier España, S.L.U. Este es un artículo Open Access bajo la licencia CC BY-NC-ND (http://creativecommons.org/licenses/by-nc-nd/4.0/).
\end{abstract}

Two-dimensional echocardiographic assessment of area and volume of the right atrium indexed to the body surface in a healthy population group

\begin{abstract}
Objective: To determine the area and normal volume of the right atrium indexing it to the body surface by means of a two-dimensional echocardiogram in a health population group in order to obtain the baseline values for future studies of unhealthy population groups.

Methods: Descriptive observational study.
\end{abstract}

\footnotetext{
* Autor para correspondencia.

Correo electrónico: gustavorestrepomd@une.net.co (J. Mosquera).
} 
Results: 64 healthy patients were included, aged 17 to 68 , who fulfilled all selection criteria. The average age was 34 years old $(S D \pm 12.19)$ and the male:female ratio was of $1: 1.78$, finding an average right atrium area of $12.2 \mathrm{~cm}^{2}(S D \pm 1.46)$, a minimum of $10 \mathrm{~cm}^{2}$ and a maximum of $16 \mathrm{~cm}^{2}$. In addition to this measurement, for this cavity the volume was also calculated, where an average of $28.7 \mathrm{~mL}$, with a minimum of $19 \mathrm{~mL}$ and a maximum of $42 \mathrm{~mL}$ (SD \pm 5.19 ) was observed.

Conclusions: This is the first study in Colombia to report normal values indexed to the body surface of the area and volume of the right atrium in a healthy population group.

(c) 2015 Sociedad Colombiana de Cardiología y Cirugía Cardiovascular. Published by Elsevier España, S.L.U. This is an open access article under the CC BY-NC-ND license (http://creativecommons.org/licenses/by-nc-nd/4.0/).

\section{Introducción}

La determinación del tamaño de la aurícula derecha mediante ecocardiografía bidimensional se considera clínicamente importante para la valoración de pacientes que sufren diferentes patologías como falla cardíaca, hipertensión pulmonar, arritmias y cardiopatías congénitas ${ }^{1}$. Numerosos estudios reportan la importancia clínica y pronóstica del volumen de la aurícula derecha, ya que este es un predictor independiente de los resultados adversos en pacientes con patologías cardiovasculares y pulmonares ${ }^{2}$. Como desventaja está la falta de estandarización de los volúmenes de la aurícula derecha, de ahí que las guías recomienden la cuantificación del área como el mejor parámetro para la valoración de dicha estructura y no indiquen la cuantificación de su volumen de forma rutinaria debido a la escasez de información estandarizada por ecocardiografía bidimensional ${ }^{2,3}$. Existen muy pocos estudios que muestren los valores normales de la aurícula derecha indexados en el área de superficie corporal en pacientes sanos ${ }^{2}$, y allí radica la importancia del estudio, ya que con esta información se obtendrían datos de vital importancia que podrían ser extrapolados a las poblaciones de grupos especiales.

\section{Metodología}

\section{Población de estudio}

Estudio observacional, descriptivo, que incluyó una población de pacientes adultos mayores de 18 años, sanos, sin ninguna patología cardiovascular asociada, elegidos dentro de los pacientes remitidos al servicio de Cardiología no invasiva para evaluación mediante ecocardiografía convencional.

Se consideraron criterios de exclusión los pacientes con patologías previas a nivel cardíaco tipo falla cardíaca, cardiopatía isquémica o enfermedad valvular, la presencia de dispositivos de estimulación cardíaca, cardiopatías congénitas, hipertensión pulmonar, ritmos diferentes al sinusal, cirugías cardíacas previas y mala ventana acústica, así como todo aquel paciente que en el estudio ecocardiográfico tuviera alguna alteración no conocida previamente.

\section{Ecocardiografía 2D}

A todos los participantes se les realizó un ecocardiograma transtorácico convencional mediante un equipo Philips IE33 con medición del área y del volumen de la aurícula derecha en el plano apical con cuatro cámaras, siguiendo las recomendaciones de las guías. La toma de las imágenes se hizo mediante sincronización con el electrocardiograma y todos estos valores fueron indexados en el área de superficie corporal.

Los estudios se almacenaron en formato digital y fueron transferidos a una estación de trabajo fuera de línea para su análisis posterior.

\section{Resultados}

En la Clínica Medellín se realizaron 1.224 ecocardiogramas durante agosto de 2012 a septiembre de 2012, obteniéndose un total de 64 pacientes entre las edades de 17 y 68 años que cumplían los criterios de selección. El promedio de edad observado fue de 34 años ( $D E \pm 12,19)$ y la razón de masculinidad fue de 1:1,78.

En cuanto a la evaluación de las medidas antropométricas se observó un promedio de peso en kilogramos de $63,52 \mathrm{~kg}$ $(\mathrm{DE} \pm 11,8)$ y una media de talla de $162 \mathrm{~cm}(\mathrm{DE} \pm 8,91)$. Con estos datos se calculó la superficie corporal, encontrando un mínimo de $1,3 \mathrm{~m}^{2}$, un máximo de $2 \mathrm{~m}^{2}$ y un promedio de $1,64 \mathrm{~m}^{2}$.

Dentro de las principales indicaciones de solicitud del ecocardiograma se observó el dolor torácico como el más prevalente, seguido de hallazgos al examen físico como el soplo cardíaco y la sensación de palpitaciones, encontrado en un $20,5,15,9$ y $11,4 \%$ respectivamente (figs. 1 y 2 ).

En la figura 3 se pueden observar otras indicaciones (figs. 4-6).

\section{Evaluación ecocardiográfica}

Se continuó con la evaluación de los diferentes parámetros de la función ventricular a través de ecocardiograma transtorácico. 


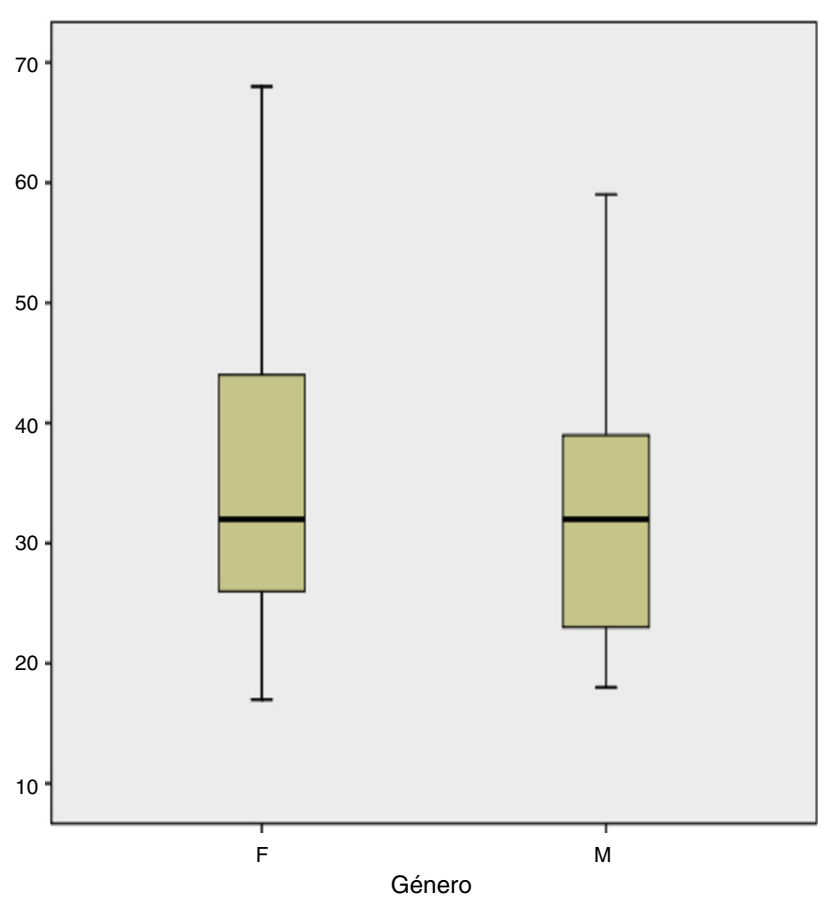

Figura 1 Edad y sexo.

El primer parámetro evaluado de la función ventricular fue la fracción de eyección del ventrículo izquierdo observando un promedio de $64 \%$ (DE $\pm 1,07 \%)$.

En cuanto a la evaluación de la aurícula derecha, se evidenció un promedio de área calculado en $12,2 \mathrm{~cm}^{2}$ (DE \pm $1,46)$ con un mínimo observado en $10 \mathrm{~cm}^{2}$ y un máximo de $16 \mathrm{~cm}^{2}$. Además de esta medición, para esta cavidad también se calculó el volumen, donde se observó un promedio de $28,7 \mathrm{~mL}$, con un mínimo de $19 \mathrm{~mL}$ y un máximo de $42 \mathrm{~mL}$ $(\mathrm{DE} \pm 5,19)$.

Otros parámetros de la función ventricular evaluados de manera cuantitativa a través de este examen fueron la excursión sistólica del anillo tricúspide (TAPSE), donde se pudo observar un promedio de $2,24 \mathrm{~cm}(\mathrm{DE} \pm 0,28)$.

De igual manera, se realizó el cálculo del diámetro basal del ventrículo derecho evidenciándose un promedio de $3,28 \mathrm{~cm}(D E \pm 0,53)$.

En la tabla 1 se describen otras mediciones de la función ventricular (tabla 2).

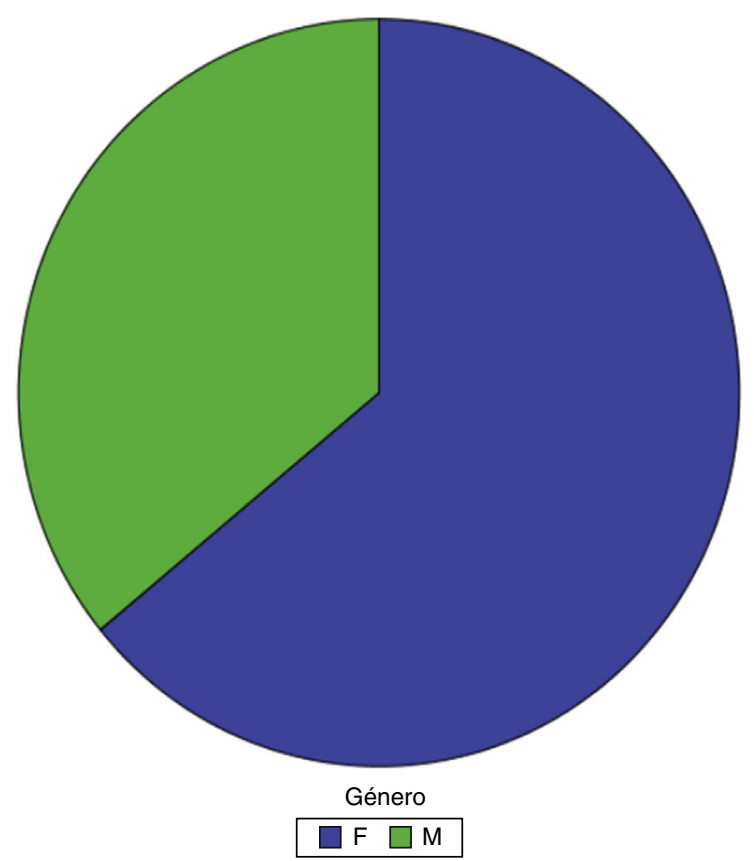

Figura 2 Distribución por género.

\section{Análisis de correlación}

Se procedió a realizar un análisis de correlación entre los diferentes parámetros observados de la función miocárdica y las medidas antropométricas, en búsqueda de posibles asociaciones. Se utilizó la prueba no paramétrica de Spearman, con la que se pudo observar una correlación estadísticamente significativa $(p<0,05)$ entre la superficie de área corporal y el área de la aurícula derecha, el volumen de la aurícula derecha y el diámetro basal del ventrículo derecho.

Todas estas correlaciones observadas fueron positivas; no obstante, su magnitud fue leve $(<0,6)$, lo que hace pensar que la superficie corporal no es la única variable que condiciona estas áreas miocárdicas y su función.

Como era de esperarse, se observó una fuerte correlación entre el volumen de la aurícula derecha y su área $(0,775)$, valor estadísticamente significativo $(p=0,000)$.

A su vez, se observó una correlación estadísticamente significativa $(p=0,001)$ entre el volumen y el diámetro basal

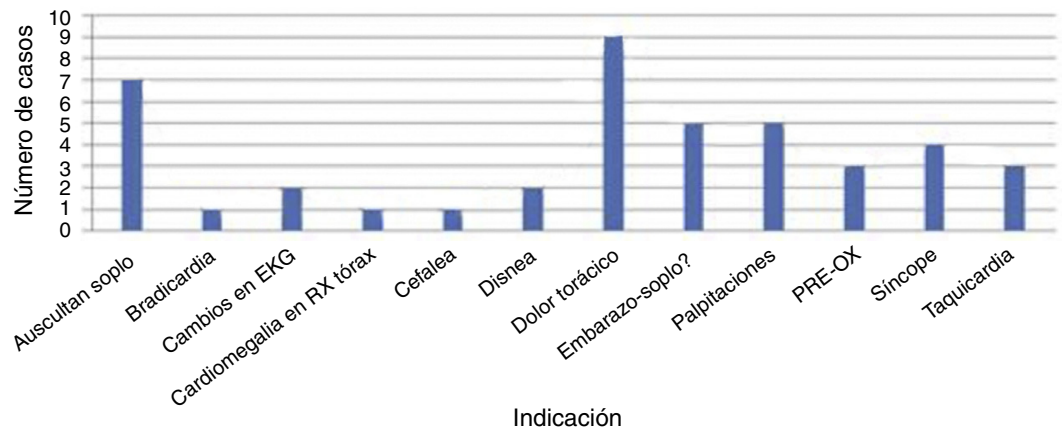

Figura 3 Distribución para indicaciones de ecocardiograma. 
Tabla 1 Principales hallazgos ecocardiográficos

\begin{tabular}{|c|c|c|c|c|}
\hline & $x$ & DE & Min. & Max. \\
\hline FEVI (\%) & $64,77 \%$ & $1,07 \%$ & $60,00 \%$ & $65,00 \%$ \\
\hline Área $\mathrm{AD}\left(\mathrm{cm}^{2}\right)$ & 12,2 & 1,46 & 10 & 16 \\
\hline Volumen $\mathrm{AD}(\mathrm{mL})$ & 28,7 & 5,19 & 19 & 42 \\
\hline TAPSE $(\mathrm{cm})$ & 2,24 & 0,28 & 1,8 & 3,3 \\
\hline Doppler tisular DTI (cm/s) & 12,77 & 1,98 & 10 & 18 \\
\hline Diámetro basal VD $(\mathrm{cm})$ & 3,28 & 0,35 & 2,8 & 4 \\
\hline PSAP (mm Hg) & 16,0 & 34,80 & 16 & 30 \\
\hline Área $A D$ index $\mathrm{cm}^{2} / \mathrm{m}^{2}$ & 7,41 & 0,92 & 5,7 & 10 \\
\hline Volumen $A D$ index $\mathrm{mL} / \mathrm{m}^{2}$ & 17,43 & 3,07 & 11,8 & 23,7 \\
\hline Diámetro medio VD index $\mathrm{cm} / \mathrm{m}^{2}$ & 1,96 & 0,25 & 1,4 & 2,6 \\
\hline
\end{tabular}

FEVI: fracción de eyección del ventrículo izquierdo; AD: aurícula derecha; TAPSE: excursión sistólica del anillo tricúspide; DTI: imagen de Doppler tisular; VD: ventrículo derecho; PSAP: presión sistólica de la arteria pulmonar.

Tabla 2 Análisis de correlación de los parámetros ecocardiográficos y las medidas antropométricas

\begin{tabular}{llll}
\hline \multicolumn{3}{c}{ Correlación de Spearman rho } \\
\hline
\end{tabular}

AD: aurícula derecha; VD: ventrículo derecho; SC: superficie corporal; FEVI: fracción de eyección del ventrículo izquierdo.

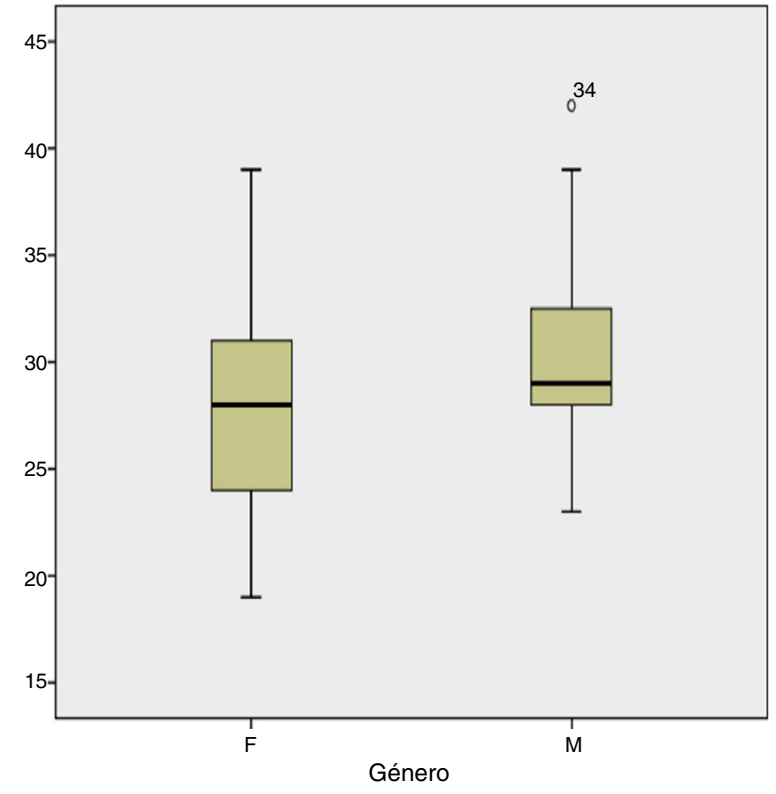

Figura 4 Volumen y sexo.

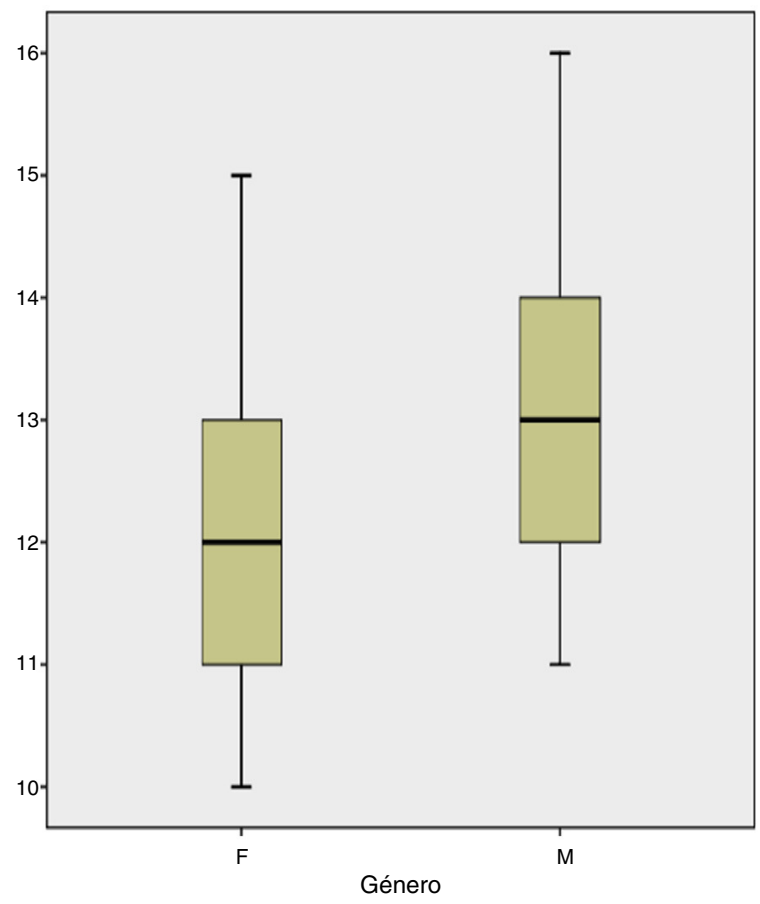

Figura 5 Área y sexo. 


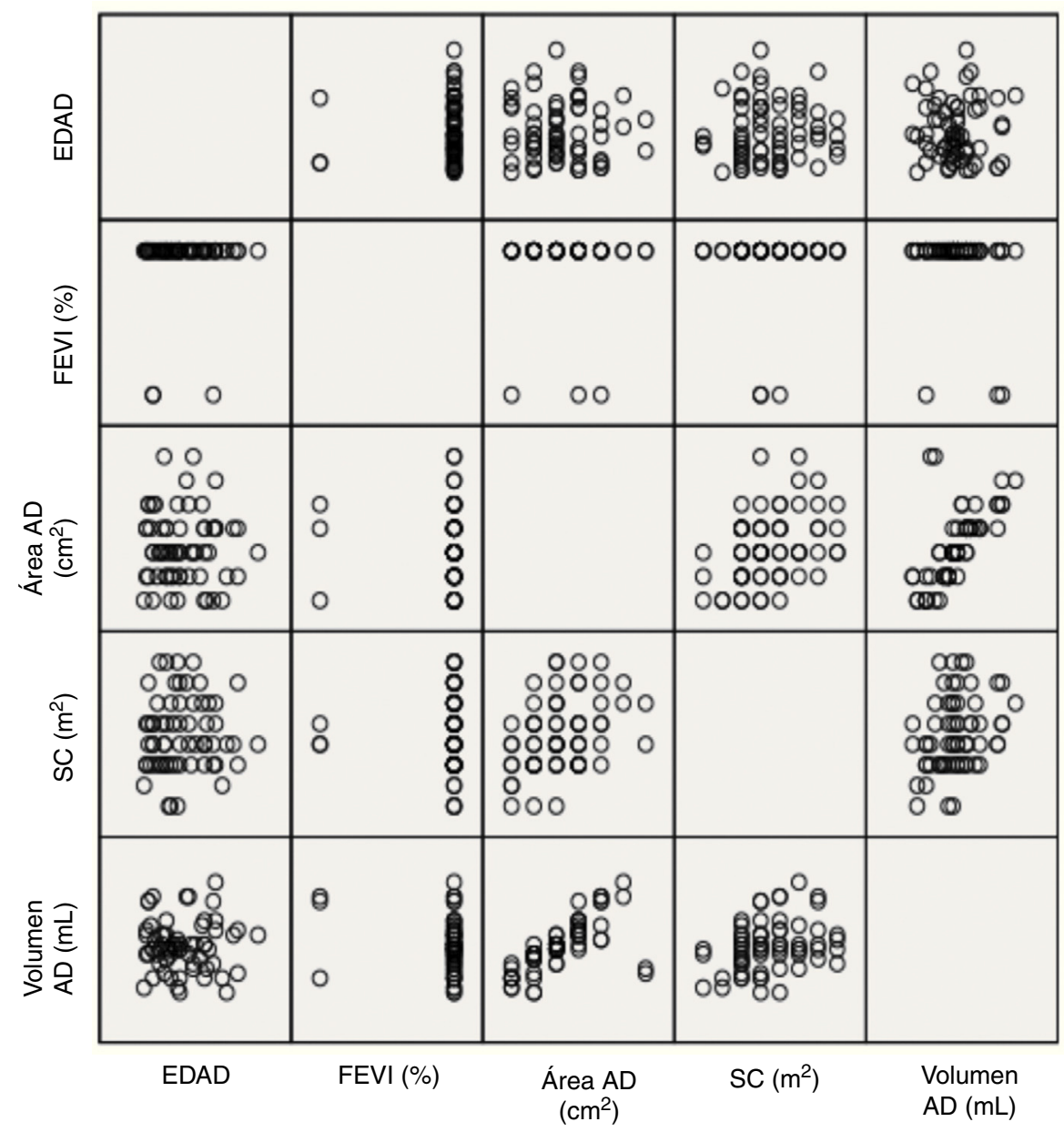

Figura 6 Correlación de los parámetros ecocardiográficos y las variables antropométricas.

del ventrículo derecho que, sin embargo, se consideró débil $(0,414)$.

No se encontró ningún tipo de correlación estadísticamente significativa entre la edad y los diferentes parámetros estudiados ni entre la fracción de eyección del ventrículo izquierdo con la función evaluada $(p>0,05)$.

\section{Comparación de medidas}

El análisis finalizó con la comparación de los promedios de cada parámetro de función ventricular por sexo, a través de la prueba no paramétrica de $U$ Mann-Whitney, en búsqueda de diferencias entre géneros en cada una de estas variables.

Como se observa en la tabla 3, existe una diferencia entre los promedios de área de la aurícula derecha entre hombres y mujeres de $(-) 1,51 \mathrm{~cm}^{2}$ (IC $\left.95 \%=-2,178 \mathrm{a}-0,852\right)$ la cual resultó ser estadísticamente significativa $(p=0,0000)$.

De igual manera, esta diferencia resultó ser estadísticamente significativa $(p=0,013)$ para los volúmenes de la aurícula derecha, donde, como en el caso anterior, se observó una diferencia de (-) 3,83 mL (IC 95\%=-5,907 a $-0,721)$.

Finalmente y en concordancia con lo observado, esta diferencia entre promedios también resultó ser estadísticamente significativa $(p=0,000)$ entre las superficies de área corporal por sexo, siendo esta a favor del sexo masculino en $0,231 \mathrm{~m}^{2}$.

\section{Discusión}

Aunque existen pocos datos en la literatura que reporten el volumen y el área de la aurícula derecha indexada en población sana mediante ecocardiografía bidimensional, hoy se sabe y se reconoce la importancia clínica de la dilatación de la aurícula derecha como predictor independiente de eventos adversos cardiovasculares en una serie de grupos especiales, como son los pacientes con hipertensión pulmonar, arritmias, enfermedades congénitas del corazón y falla cardiaca $^{4-6}$.

Existen algunos estudios que demuestran que la ecocardiografía bidimensional puede subvalorar los volúmenes obtenidos de la aurícula derecha ${ }^{8,10}$, fenómeno que se explica muy probablemente por la forma irregular y la geometría compleja de dicha cavidad ${ }^{9,10}$, lo que a su vez se ha demostrado mediante ecocardiografía tridimensional y resonancia magnética de corazón ${ }^{8,9}$. Este hecho se considera como limitación del estudio ya que no se tienen datos que comparen nuestros resultados con otras modalidades de imágenes diagnósticas. A pesar de estas limitaciones, estos resultados pueden establecer datos de referencia del 
Tabla 3 Análisis bivariado entre parámetros ECO, SC y género

\begin{tabular}{|c|c|c|c|c|c|c|c|}
\hline \multicolumn{8}{|c|}{ Análisis bivariado sexo, parámetros ecocardiográficos } \\
\hline & \multirow[b]{2}{*}{ Género } & \multirow[b]{2}{*}{$\mathrm{N}$} & \multirow[b]{2}{*}{$\mathrm{X}$} & \multirow[b]{2}{*}{$\mathrm{DE}$} & \multirow[b]{2}{*}{$\mathrm{P}$} & \multicolumn{2}{|l|}{ IC $95 \%$} \\
\hline & & & & & & Inf. & Sup. \\
\hline \multirow[t]{2}{*}{ Área $A D\left(\mathrm{~cm}^{2}\right)$} & $\mathrm{F}$ & 41 & 11,66 & 1,23 & 0 & $-2,17$ & $-0,85$ \\
\hline & M & 23 & 13,17 & 1,33 & & & \\
\hline \multirow[t]{2}{*}{ Volumen AD (mL) } & $\mathrm{F}$ & 41 & 27,51 & 4,87 & 0,01 & $-5,90$ & $-0,72$ \\
\hline & M & 23 & 30,83 & 5,1 & & & \\
\hline \multirow[t]{2}{*}{ Área $A D$ index $\mathrm{cm}^{2} / \mathrm{m}^{2}$} & $\mathrm{~F}$ & 41 & 7,36 & 0,76 & 0,55 & $-0,62$ & 0,33 \\
\hline & M & 23 & 7,50 & 1,16 & & & \\
\hline \multirow[t]{2}{*}{ Volumen $\mathrm{AD}$ index $\mathrm{mL} / \mathrm{m}^{2}$} & $\mathrm{~F}$ & 41 & 17,35 & 2,76 & 0,78 & $-1,83$ & 1,39 \\
\hline & M & 23 & 17,57 & 3,63 & & & \\
\hline \multirow[t]{2}{*}{$\mathrm{SC}\left(\mathrm{m}^{2}\right)$} & $\mathrm{F}$ & 41 & 1,58 & 0,14 & 0 & $-0,25$ & $-0,09$ \\
\hline & $M$ & 23 & 1,76 & 0,16 & & & \\
\hline
\end{tabular}

AD: aurícula derecha; SC: superficie corporal.

volumen y del área de la aurícula derecha obtenidos de pacientes sanos, los cuales podrían ser extrapolados a pacientes con patologías cardiovasculares y pulmonares establecidas $^{1,2}$, pudiéndose estratificar el riesgo de mortalidad y determinar valores pronósticos, así como documentar remodelado reverso posterior a la ablación de arritmias auriculares ${ }^{2}$.

En cuanto a la evaluación de la aurícula derecha, en los resultados del estudio se evidenció un promedio de área calculado en $12,2 \mathrm{~cm}^{2}(\mathrm{DE} \pm 1,46)$ con un mínimo observado en $10 \mathrm{~cm}^{2}$ y un máximo de $16 \mathrm{~cm}^{2}$. Además de esta medición, para esta cavidad también se calculó el volumen, donde se observó un promedio de $28,7 \mathrm{~mL}$ ( $D E \pm 5,19)$. Además, se observó que el tamaño de la aurícula derecha se relacionó débilmente con la superficie corporal, la cual pudiera no ser la mejor forma de indexar el volumen y el área de la cavidad, de modo que la altura elevada a la 2,7 podría ser la mejor forma de indexar estos valores y tenga mayor correlación con la superficie corporal.

\section{Conclusiones}

Este es uno de los primeros estudios que reporta el valor de referencia indexado del volumen y del área de la aurícula derecha por medio de ecocardiografía bidimensional en una población sana, hecho que tiene una implicación clínica importante en la valoración de grupos de poblaciones especiales con enfermedades cardíacas y pulmonares ${ }^{1,5}$, mejorando así el entendimiento de los diferentes mecanismos implicados en la remodelación de la aurícula derecha ${ }^{6}$, así como en la identificación de pacientes con pronósticos clínicos adversos ${ }^{7}$.

Una limitación del trabajo es la ausencia de comparación con otras técnicas diagnósticas como con la ecocardiografía $3 \mathrm{D}$, que, al no requerir de modelos geométricos, los datos obtenidos podrían ser más reproducibles que los realizados por ecocardiografía bidimensional, así como con resonancia magnética de corazón, considerada como el estándar de oro en la valoración de cavidades derechas.

\section{Conflicto de intereses}

Los autores declaran no tener ningún conflicto de intereses.

\section{Bibliografía}

1. Moreno J, Pérez de Isla L, Campos N, Guinea J, DomínguezPérez L, Saltijeral A, et al. Right atrial indexed volume in healthy adult population: reference values for two-dimensional and three-dimensional echocardiographic measurements. Echocardiography. 2013;0:1-5.

2. Quraini D, Pandian N, Patel AR. Three-dimensional echocardiographic analysis of right atrial volume in normal and abnormal hearts: comparison of biplane and multiplane methods. Cardiovascular Imaging and Hemodynamic Laboratory, Tufts Medical Center, Boston, Massachusetts. Echocardiography. 2012;29:608-13.

3. Rudski L, Wyman C, Afilalo J, Lanqi H, Handschumacher M, Chandrasekaran K, et al. Guidelines for the echocardiographic assessment of the right heart in adults: a report from the American Society of Echocardiography endorsed by the European Association of Echocardiography, a registered branch of the European Society of Cardiology, and the Canadian Society of Echocardiography Montreal, Quebec, Canada; New York, New York; Boston, Massachusetts; Phoenix, Arizona; London, United Kingdom; San Francisco, California. J Am Soc Echocardiogr. 2010;23:685-713.

4. Sallach JA, Tang W, Borowski A, Tong W, Porter T, Martin MG, et al. Right atrial volume index in chronic systolic heart failure and prognosis. J Am Coll Cardiol Img. 2009;2:527-34.

5. Yildirimturk O, Tayyareci Y, Erdim R, Ozen E, Yurdakul S, Aytekin $\mathrm{V}$, et al. Assessment of right atrial pressure using echocardiography and correlation with catheterization. J Clin Ultrasound. 2011;39:337-43.

6. D’Ascenzi F, Cameli M, Padeletti M, Lisi M, Zaca V, Natali B, et al. Characterization of right atrial function and dimension in top-level athletes: a speckle tracking study. Int J Cardiovasc Imaging. 2014;7:222-9.

7. Tsang TS, Abhayratna WP, Barnes ME, Miyasaka Y, Gersh BJ, Bailey KR, et al. Prediction of cardiovascular outcomes with left atrial size: Is volumen superior to area or diameter? J Am Coll Cardiol. 2006;47:1018-23.

8. Rudski LG, Lai WW, Afilalo J, Hua L, Handschumacher MD, Chandrasekaran K, et al. Guidelines for the 
echocardiographic assessment of the right heart in adults: A report from the American Society of Echocardiography. J Am Soc Echocardiogr. 2010;23:685713.

9. Lang RM, Badano LP, Tsang W, Adams DH, AgricolaE, Buck T, et al. Recommendations for image acquisition and display using three-dimensional echocardiography., FASE. J Am Soc Echocardiogr. 2012;25:3-46.

10. Keller AM, Gopal AS, King DL. Left and right atrial volumen by freehand three dimensional echocardiography: In vivo validation using magnetic resonance imaging. Eur J Echocardiogr. 2000;1:55-65. 